\title{
Leitura, literatura e tradução: a necessidade de adequações no ensino de línguas não-maternas
}

Sergio Flores Pedroso

Universidade Federal do Mato Grosso

\begin{abstract}
Neste artigo, a leitura em línguas não-maternas é abordada pela via do lugar que a literatura ocupa em livros didáticos de espanhol e, mais concretamente, através do gênero narrativa. A interpretação e a consideração da tradução no processo de ensino de línguas não-maternas em geral e da leitura em particular fazem parte dos aspectos problematizados em torno da relação literatura/ ensino. Isso porque a premissa de que deriva este trabalho é a de que a língua materna gerencia toda aproximação de outra língua, sendo que isso envolve os componentes ideológicos da linguagem.

This paper looks at reading in foreign languages from the place that textbooks of Spanish as a foreign language attribute to literature, especially through the narrative genre. Interpretation and translation are part of the aspects focused on. Two processes, the one of learning and the one of reading, are included within the link between literature and learning. That is because the premise of this paper is that the mother tongue is constituent of the process of foreign language learning. The ideological components of language play a definite role in it.
\end{abstract}

\section{Introdução}

A leitura está longe de ser assunto esgotado na pedagogia das línguas não-maternas (LNM). O interesse em sua abordagem e reformulações parece dever-se a considerações de vários tipos, que atravessam os questionamentos que são feitos ao processo de ensino no que diz respeito ao sujeito, ao processo cognitivo em si, ao conceito de linguagem, de texto, à participação do social - história, ideologia, cultura - e a tantos outros aspectos considerados participantes da construção do conhecimento.

A leitura e o tratamento que o ensino de LNM lhe dá, mediado pelos livros didáticos (LD), serão discutidos a seguir. O debate será feito à luz das concepções dos termos mencionados no primeiro parágrafo 
e da leitura e da literatura propriamente ditas. Uma abordagem assim envolve desdobramentos que, por sua vez, levam ao esboço de uma proposta de trabalho com o literário em LNM, concretamente no ensino de espanhol, cuja explicitação foi incluída na parte de análise de dados deste artigo.

\section{Leitura, literatura e tradução}

A leitura em LNM é assumida neste artigo como processo interpretativo cuja base é contrastiva. Não se trata, contudo, do contrastivo que, na história do ensino de LNM, se justificava como expediente para determinar os aspectos de afastamento e de maior proximidade entre as línguas, em função da antecipação de eventuais resistências à aprendizagem. Essa concepção, segundo Dabène (1998, p. 393), era a de que as dificuldades de aprendizagem dependiam exclusivamente de tal afastamento ou proximidade.

Trata-se, isto sim, de uma concepção do contratisvo em que a língua materna (LM) é considerada constitutiva de toda aproximação de uma LNM porque ela é fundadora da estruturação psíquica do sujeito (REVUZ, 1998, p. 217). A LM não é instrumento, é o lugar inaugural do saber e através dela se reorganizam e adaptam os saberes em outras línguas, numa base em que o componente sócio-histórico é o fundamento do caráter relacional que constitui a abordagem de toda LNM.

A relação que se estabelece com a LNM no processo de aprendizagem se dá num percurso de idas e vindas que torna possível o desestranhamento de um modo de significar diferente. A pertinência desse fluxo entre um dentro e um fora da língua primeira é justificado pela perspectiva de utilização desse modo de significar respeitando estratégias de formulação que se apresentam padronizadas e fazem parte, por isso, do repetível.

As maneiras comuns de formular são facilmente detectáveis quando na sua observação prevalece a forma. Entretanto, o comum é que a enunciação se processe num patamar de implícitos apenas inteligíveis para o conjunto de usuários da língua em que ela se desempenha como primeira ou materna. A via pedagógica, um longo período de imersão ou ambas as condições conjugadas têm se 
apresentado como o expediente que supre - sempre parcialmente ${ }^{1}-$ esse determinante fator identitário coletivo.

A literatura, como lugar por excelência do trabalho lúdico com a linguagem (ORLANDI, 1983, p. 85), é exemplar nesse equacionamento de implícitos, em função da produção de sentidos visando ao estético. Nesse processo, os elementos constitutivos vernáculos são prevalecentes no processo de construção de sentidos. Os elementos valorativos que constituem esse processo estão localmente delimitados pela geografia, a classe ou o grupo social e as representações de si e do que essa classe ou grupo considera heterogêneo. Esse modo de significar está determinado pela história e pela maneira como ela se explicita no plano social como prática: a ideologia e a cultura.

Os elementos constitutivos acima referidos e os pontos de ancoragem geográfico, grupal e imaginário constituem o espaço em que se produz o discurso, cujos limites se caracterizam pela mobilidade. Tal espaço resulta de um processo contraditório de reformulaçãoparáfrase cuja pertinência é dada pela existência de um nexo discursivo que se desempenha como dominante (PECHEUX, 1988; SERRANIINFANTE, 1998). Dentro desse espaço, a que se chama formação discursiva, encontram-se as marcas que tornam possível o auto e mútuo reconhecimento identitário através da linguagem e a partir do qual todo trabalho com o simbólico é processado.

Assim, o esforço por acessar uma construção diferente de sentidos não tem como contornar o recurso à língua primeira para sua execução. A LM está na base de todo esforço de naturalização/adequação para que o recorte discursivo em LNM possa existir como acontecimento significante. Isto, quer seja privilegiando o aspecto perceptivo do uso lingüístico - a interpretação (leitura e compreensão auditiva) -, quer seja focalizando o seu lado produtivo: a produção oral e escrita.

A comparação que visa às equivalências apresenta-se, assim, como recurso expedito, produtivo e único. A paráfrase é, por isso, o recurso a partir do qual as equivalências são construídas e, em conseqüência, torna visível o processo interpretativo. Até hoje, esforços por desabonar

\footnotetext{
${ }^{1}$ Isso porque o recorte desse todo de valores conceituais e equacionamentos formais a que se teve acesso não faz parte da inscrição primeira do sujeito na linguagem, dos modos de dizer e dos efeitos emocionais que decorrem deles.
} 
ou ignorar o contrastivo têm conseguido ser relativamente eficazes em se impor. Os do primeiro tipo têm sido feitos pela via de uma quase demonização quando a língua primeira - aqui considerada referência indispensável na aproximação das LNM - se explicita nas práticas pedagógicas. Os que desconsideram o relacional entre as línguas esforçam-se em reproduzir o irrepetível: o processo de inscrição do sujeito na língua materna.

A prática discursiva genérica que este trabalho enfoca é a leitura. Através da sua abordagem, acessam-se mecanismos processuais que a constituem e conformam o que se conhece como interpretação (ORLANDI, 1996, p. 21). A essência do processo interpretativo, no que tange às LNM, é a comparação a que tenho feito referência. A esse aspecto soma-se a literatura como foco de interesse. Sua singularidade, para os efeitos da vertente discursiva dos estudos da linguagem, consiste no trabalho com o particular que imprime identidade (MILLER apud KRAMSCH, 1993, p. 131). A literatura estrangeira apresenta chaves que permitem acessar as diferenças individual e coletiva. O seu processamento se dá através de um esforço comparativo em busca de uma certa transparência favorável à interpretação.

A escrita oferece duas possibilidades que são próprias dos mecanismos antecipatórios que operam no uso da linguagem (ONG, 1982), tanto nas LNM quanto na LM: o retorno à horizontalidade do texto e o recurso à transversalidade intertextual com uma disponibilidade de tempo que a oralidade não concede. No caso da leitura em LNM, essas duas possibilidades se processam e assentam na língua de inscrição primeira do sujeito. É por isso que se trata de um processo contrastivo e necessariamente feito nos moldes da tradução.

A tradução é concebida aqui como processo interpretativo de atribuição de sentidos, que evidencia para o aprendiz os percursos de idas e vindas de uma língua à outra dentro do processo de tornar significativo numa língua o que foi enunciado a partir de valores que, próximos ou distantes, têm como característica o fato de serem diferentes porque provêm de uma prática social que se assenta sobre outro recorte do real.

Autores de filiações teóricas distantes entre si na concepção da linguagem, porém, não desconsideram de maneira alguma a tradução como interpretação e adequação. São os casos - e vou apenas colocar 
exemplos - de aqueles que consideram a linguagem como código entre os quais se encontram Jakobson (1971), Mounin (1975), Nida (1993) e Larson (1998).

Entre os pesquisadores que consideram que a linguagem existe por e para a construção de sentidos - e por isso focalizam a textualidade -, encontram-se Steiner (1978), Catford (1980), Arrojo (1986) e Venuti (1994).

O caráter de ferramenta interpretativa principal na relação entre línguas não tem impedido, contudo, que a tradução continue sendo estigmatizada por inscrições teóricas que usufruem de hegemonia no ensino de LNM. A tradução, apesar disso, prossegue subsidiando oculta e democraticamente o processo interpretativo - o daqueles que lhe reconhecem o mérito e o daqueles que lhe ignoram a função - na relação que se estabelece com as LNM.

Não é a tradução o recurso mais importante que a ferramenta contrastiva fornece. Antes, é através dela que se dá o processo contrastivo que constitui a interpretação de um texto numa língua que não é materna ou primeira. Note-se que falo em interpretação de um texto numa lingua e não em interpretação numa língua, que significaria endossar uma idéia limite que tem balizado a história do ensino de LNM. Trata-se da idéia que defende a possibilidade de se conseguir que os estudantes pensem numa LNM.

O que aqui está sendo fundamentado é o papel protagônico da LM na aproximação de outra língua, quando essa aproximação é feita a partir de um patamar concebido além da forma, como é próprio da concepção discursiva da linguagem. Dessa perspectiva, a abordagem da linguagem é feita considerando-a menos como forma do que como substância ideológica, cultural, histórica e, por isso, socialmente constituída.

Os aspectos acima são também os que gerem as antecipações possíveis - leiam-se as interpretações - e as comparações necessárias com o que se conhece do outro modo de simbolizar. Por isso é preciso levar em conta que os sentidos não são construídos sem operar com valores e estes resultam de práticas sociais com histórias diferenciadas. Esse arcabouço teórico torna produtivo o trabalho didático com a literatura no ensino de LNM em direções que, no trabalho com a abordagem comunicativa, não têm sido muito freqüentes.

A abordagem comunicativa tem se mostrado tão atrelada às propostas do LD que - como comenta Grigoletto (1999, p. 68) - 
professores e alunos se encontram unidos no papel de usuários dessa espécie de programa paralelo. Acompanhando a massificação do acesso ao ensino de LNM, o LD tem suprido necessidades do professor no seu desempenho, ganhando protagonismo e tornando-se indispensável no processo pedagógico.

A influência do LD tem atingido tal alcance, que é referência para determinar o que, como e quando ensinar. Ele homogeneíza o público, comuniza interesses e desconsidera o principal lugar do heterogêneo na pedagogização de uma relação entre línguas que dá prioridade ao social através do (inter)cultural. Considerar criticamente o LD, por isso, não é pouco importante. Ele representa o projeto maior onde todos os materiais estão articulados em função da sua proposta de prática pedagógica. Essas suas características justificam a necessidade de abordá-lo criticamente.

No caso do ensino de espanhol, a presença da literatura nesses materiais didáticos se dá principalmente através da narrativa como gênero, o que não exclui outros, dentro do mostruário que apresentam. Isso, no meu parecer, deve-se ao fato de que a narrativa aparenta se afastar menos do uso comum das línguas, o que é sustentado pelo pressuposto de transparência da linguagem, da remissão direta da linguagem ao mundo. Esse parece ser um dos motivos da recorrência de recortes desse gênero. Entretanto, um dos traços de maior relevo do literário é o uso da linguagem diferentemente do comum.

Em geral, o recurso ao literário nos LD parece envolver três propósitos: ilustra o uso da língua do ponto de vista formal, exercita a interpretação de textos (concebidos) numa $L N M$, por via de regra apagando o componente tradutório, e representa uma parcela do conteúdo "cultural" em rigor com a tradição do ensino de LNM e, mais recente e paradoxalmente, com a inscrição comunicativa. ${ }^{2}$

Nesta primeira parte do artigo, foram feitas explicitações teóricas que embasam a análise da proposta de trabalho com a leitura de recortes

\footnotetext{
${ }^{2}$ Nesse sentido, Valdés (1986, p. 137) comenta: "a afirmação de que a literatura pode ser usada para ensinar cultura é provavelmente tão amplamente aceita que se transformou em clichê". Tal questionamento deve-se a que ele defende que literatura e leitura não são idênticas, como é tendência considerar no ensino de LNM.
} 
literários em três LD em uso no Brasil. Essa crítica irá funcionar como ponto de partida para esboçar aspectos que considero necessários levar em conta, com esse mesmo propósito, dentro de uma abordagem discursiva da linguagem.

Os LD sob análise - Ven, Cumbre e Punto Final, níveis básico, intermediário e superior, respectivamente - foram escolhidos por quatro razões:

- fazem parte do grupo dos LD usados no Brasil em centros de línguas de universidades públicas em que verifica-se a inexistência de um título único em programas de ensino de espanhol;

- são exemplos a mais dos muitos títulos para o ensino da língua espanhola como estrangeira que a "indústria" de LD da Espanha colocou no mercado brasileiro sob seu controle;

- os três níveis de complexidade lingüística possibilitam uma maior noção da assunção teórica prevalecente no trabalho com textos de leitura no ensino de espanhol para estrangeiros;

- todos incluem recortes literários entre seus materiais de leitura.

A seguir, passo a mostrar como é apresentado o trabalho com a leitura a partir de trechos literários incluídos em cada um dos livros escolhidos.

\section{Os dados e sua adequação}

O traço didático que vincula os três títulos escolhidos não se afasta do comum das publicações metodológicas para o ensino de LNM: a inscrição cognitivista que se reflete na ênfase gramatical na organização do material didático. Isso é da maior importância para achar neles a pertinência do esforço por introduzir explicitamente nomenclatura gramatical - o que ressalta a sua atenção ao formal da linguagem - para torná-la operativa pelo público-alvo mediante a manipulação mecanicista de seus elementos através de exercícios de reconhecimento e de substituição. Ilustro abaixo este comentário com exemplos de cada título:

1. Haz una lista de todos los verbos que aparecen en el texto de la encuesta anterior. Luego escribe las formas del pretérito indefinido de cada uno de ellos, clasificándolas en formas regulares y formas irregulares (Cumbre, p. 2). 
2. Subraya e identifica en estas oraciones todos los pronombres que actúen como complemento de objeto directo o indirecto (Punto Final, p. 68).

3. En parejas. A pregunta y B responde negativamente:

- ¿Ha llamado alguien por teléfono?

- No, no ha llamado nadie (Ven, p. 127).

O comentário inicial e os exemplos acima claramente se referem a aspectos fora do âmbito da leitura e do literário. Julguei pertinente introduzi-los neste lugar porque se trata do fundamento de todo trabalho didático que cada volume propicia com a LNM e que não exclui a sua proposta com a leitura, a literatura e a consideração da LM através da tradução.

Assim, a partir do pressuposto do sentido dado, o lugar atribuído à interpretação e, por isso, ao contrastivo, reduz-se ao meramente formal, em função de dois fatores:

a) a gramática

Sustituye los verbos conjugados en presente por formas verbales de pasado. Discute los valores de los tiempos.

Matilde Urrutia, mi mujer

Mi mujer es provinciana como yo. Aunque esto no interesa a nadie, somos felices. Dividimos nuestro tiempo en común en largas permanencias en la solitaria costa de Chile. No en verano, porque el litoral reseco por el sol se muestra entonces amarillo y desértico. Sí en invierno, cuando en extraña floración se viste con las lluvias y el frío, de verde y amarillo, de azul y de purpúreo. Algunas veces subimos del salvaje y solitario océano a la nerviosa ciudad de Santiago, en la que juntos padecemos con la complicada existencia de los demás (...).

Confieso que he vivido, Pablo Neruda (Punto final, p. 18).

b) a recorrente atenção intradiscursiva mediante o que, quem, quando, onde, como e por quê

Lee atentamente y responde de manera corta según lo que entendiste: ¿qué?, ¿quién?, ¿dónde? 
...Así transcurrió el tiempo y llegó el que había sido señalado para la boda. La casa de los Reinoso andaba toda revuelta con los preparativos que hacían. Una cuadrilla de artesanos pulía los suelos. (...) La modista iba y venía, casi a diario, a probar a la desposada las prendas del ajuar, las vecinas acudían a curiosear las novedades y en las sobremesas de la familia no se hablaba sino de las familias que debían asistir a la boda clasificándolas cuidadosamente en las dos categorías de padrinos y simples invitados (...).

El cuarto de enfrente, Rómulo Gallegos (Ven, p.73).

Desatendem-se, assim, singularidades locais e individuais da língua nos planos intradiscursivo - da ordem do formulativo - e interdiscursivo, da ordem do histórico-ideológico. A partir dessas singularidades, o tradutório-contrastivo ganha dimensão discursiva. Os exercícios em que a LM serviria de ferramenta semântica e enunciativa, quando incluídos, respondem a uma assunção da linguagem de que o histórico e o ideológico não participam. Eles limitam-se a:

1. listas de palavras:

En parejas: ¿cómo se dice en vuestro idioma?

Teja, tejado, balcón, fachada, ladrillo, techo, reja, puerta de entrada, cristales, jardineras, portal. (Cumbre, p. 38),

2. orações subordinadas em ausência das principais:

a) Traduce a tu idioma las siguientes frases:

...aunque muchas de ellas sean desconocidas...

...aunque muchas de ellas no hayan sido descubiertas

b) Observa especialmente cómo has traducido los verbos. ¿Coinciden los tiempos verbales en español y en tu idioma? (Cumbre, p. 58)

3. palavras e expressões idiomáticas e formulaicas:

a) Explica en español el significado de:

Vale la pena, estoy satisfecha, relaciones personales, adecuada para, empresarias, jefa, me defiendo bien, presenta problemas.

b) Traduce esas mismas palabras a tu idioma. (Cumbre, p. 67). 
4. orações num tempo verbal determinado:

a) Traducid a vuestro idioma estas oraciones:

Dudo del futuro.

Parece que Leonardo no llegó a construir un modelo útil.

Yo pensaba que la bicicleta era una invención de la tecnología hispana.

Dudo que la bicicleta sea un invento español.

Pero ahora creo que todos saben montar en bici.

b) Comparad el tiempo del verbo español que aparece en cursiva y el tiempo del verbo en vuestra lengua. (Cumbre, p. 121).

Tudo parece evadir o textual, que é como se desempenha naturalmente a linguagem. A estruturação dos LD em questão é escorada por conceitos claramente estabelecidos - embora não explicitados - de linguagem (transparente, sentido dado) e de sujeito (intencional). Deles resultam outros não menos importantes, como o de interpretação, tradução, cultura e literatura e o lugar que os LD propõem que esses conceitos ocupem no processo pedagógico. Funcionando de modo sistêmico, eles imprimem coerência às propostas do material didático, independentemente do seu compromisso teórico.

A partir da consideração acima, impõe-se a pergunta: por e para que a literatura nessas condições? Certamente uma das respostas possíveis não envolve a familiarização com contextos histórico-sociais, regularidades formulativas, diferenças dialetais ou valores estéticos, através dos quais a literatura deveria ser explorada no ensino de LNM.

A resposta parece encontrar-se quando se recua àquilo que justificava o ensino de LNM, passando pelo método dito de gramática e tradução e, já na modernidade, pelo ensino com viés "civilizatório", em que cultura era arte e o conhecimento - mesmo que fosse apenas de nomes de obras e autores - dava prestígio e, por isso, facilitava a promoção social. Amostras de literatura eram disponibilizadas nos LD com efeitos que potenciavam o fascínio pelos grandes centros de "cultura", coerentemente com o colonial na linguagem, e um exemplo da exploração política do imaginário pela via "asséptica" da "cultura".

Assim, nos três títulos que estão sendo abordados, o literário parece ter sido introduzido para oferecer um mostruário de obras e 
autores. Em Cumbre, para citar apenas um título, são introduzidos trechos mínimos de obras sobre as quais nem sequer são apresentadas sinopses. Nesse livro, estão presentes autores como García Márquez, Camilo José Cela, Luis Racionero, Luis Sepúlveda, Isabel Allende, Luís Landero, Cabrera Infante, Miguel Angel Asturias, Angeles Mastretta, Alfredo Bryce Echenique, Eduardo Mendoza, Laura Esquivel, Gerardo Diego e Rafael Alberti.

Esse aglomerado de recortes literários de diversos países e regiões tem como efeito o favorecimento da idéia de um pretenso universalismo da língua em que não contam as diferenças entre as vertentes oral e escrita e ainda menos as variantes dialetais. Nessa mesma ordem de idéias, em se tratando da escrita, o literário é apresentado como prova (forjada) da existência de um espanhol genérico no uso estético da língua.

Privilegiar a forma - e a proposta pedagógica de cada um dos três volumes em foco parece demonstrar isso - significa não considerar que os sentidos são produzidos e que a ideologia e a cultura participam desse processo, sustentando e atravessando todo o trabalho com a linguagem. Em conseqüência de tal "esquecimento", a interpretação reduz-se ao estabelecimento de equivalências codificadas em que se desconsidera o heterogêneo histórico - próprio do discurso (ORLANDI, 1996) - e o heterogêneo individual - próprio da arte (ADORNO, 1999) -, ambos presentes no texto literário.

Exercícios que enfocassem em separado, na medida do possível, o trabalho com a linearidade formulativa e com a construção argumentativa, respectivamente, poderiam atrair a atenção para diferenças formais e discursivas entre as línguas. O trabalho com esse funcionamento que, artificial e momentaneamente, foi desunido, contribuiria para estimular a atitude analítica dos estudantes a respeito do discursivo em ambas as línguas, com exercícios em que o formal e o argumentativo se conjugam, como se dá naturalmente o discurso.

Assim, exercícios como os que elenco abaixo a partir do recorte do texto Confieso que he vivido, de Pablo Neruda, cumpririam o objetivo de conseguir enunciação e sensibilização ao discurso a partir do trabalho com o argumentativo, com o formulativo e com a conjunção de ambos. Tudo considerando o importante papel que a LM desempenha no processo. Os exercícios são os que seguem: 
1) Explica qué no le gusta de Santiago a la pareja.

2) ¿Cómo el poeta se refiere a las flores y al crecimiento de plantas en invierno?

3) Di eso a tu manera en español.

4) Traduce al portugués las palabras del escritor.

5) Escribe eso en portugués corriente.

6) Refiérete con tus palabras en español y resumidamente a lo que dice el párrafo.

7) Esta es una traducción al portugués de ese libro. Lee la traducción de las oraciones que hiciste y la comercial. Vamos a discutir las diferencias.

8) Trata de narrar en español en el papel lo mismo que cuenta el escritor, pero de forma sencilla. Préstales atención a las diferencias.

9) Observa el lugar donde aparecen palabras como largas, solitaria, extraña. El español pone el adjetivo antes del nombre cuando quiere resaltar la calidad, o después, con el mismo objetivo, cuando es usual ponerlo antes. Invierte la posición de los adjetivos en el texto y te ayudo a notar el efecto

10) ¿Cómo es en portugués? Muéstrame ejemplos de este libro ( periódico o revista).

11) Refiérete, en español, a calidades en objetos y cualidades en personas, resaltándolas o no.

12) Fíjate que la palabra nosotros no se usa en el texto. En español no es necesario poner o decir yo, tú, él, etc., porque el verbo y otras palabras identifican de qué persona se trata. ¿Qué verbos y qué palabras identifican a ese nosotros?

13) Traduce al portugués las tres primeras líneas y fíjate si usas o no el pronombre.

Os exercícios 1, 2, 3, 4, 5 e 6 foram concebidos para avaliar a compreensão a partir do fio argumentativo, que inclui a textualidade própria do recorte. A formulação - o trabalho com a materialidade lingüística - foi abordada nos exercícios 9, 10, 11, 12 e 13. Já os exercícios 7 e 8 , representam a conjunção do formal e do argumentativo, visando trabalhar com a sensibilidade ao discurso a partir do contrastivo.

Um dos aspectos que a meu ver é uma cobrança dos recortes literários quando introduzidos no ensino de LNM é a sua distinção formal na enunciação. Nisso se condensa uma parcela importante de 
sua identidade e, no entanto, na prática pedagógica é pouco considerado. Nos exercícios apresentados aqui, chama-se a atenção para características do discurso literário, o que também envolve destacar especificidades da enunciação ordinária. Isso é feito através da ferramenta contrastiva, a tradução que está presente no processo todo de sensibilização discursiva.

\section{Considerações finais}

O recurso à literatura a partir de uma inscrição discursiva obriganos a operar com aspectos e a considerar conceitos que, no mínimo, não são priorizados em outras abordagens. No caso dos três LD que serviram de fonte de dados, a presença de recortes literários não demonstrou praticidade à própria luz dos objetivos da abordagem comunicativa, dois dos quais são a perspectiva intercultural do ensino e o fato de que os estudantes devem aprender a LNM de uso quotidiano. Curiosamente, nesse sentido, o recurso a trechos de peças de teatro e telenovelas, que se apresentam como o meio mais produtivo, é bem menos freqüente. Com eles, se reproduziria a interação em função textual - tornando visível a construção de efeitos de sentido e embora respondendo à ordem da escrita, representam o registro do oral, priorizado pelas inscrições teóricas contemporaneamente atuantes.

Sou de parecer que a legitimação do literário na leitura, dentro do processo de ensino de LNM, passa necessariamente pela atenção às condicionantes sócio-históricas - a ideologia, a cultura - em função de facilitar o acesso a uma construção de sentidos que opera com o que se apresenta como heterogêneo. A interpretação, aspecto que é o implicitamente mais valorizado para a produção discursiva, encontra na ferramenta tradutória o seu principal ponto de apoio. Trata-se de assumir o contrastivo - será que é possível sem tradução? - como a alavanca que potencializa a aprendizagem.

No raciocínio que até aqui apresentei, está fora de questão considerar - em termos de certo ou errado - o que na área já foi feito ou que agora mesmo está se processando. Trata-se, isto sim, de apresentar uma proposta de trabalho com as LNM de um modo diferente do tradicional, - nos seus pressupostos e nos seus procedimentos -, levando em consideração um dos conceitos de maior desdobramento na pós- 
modernidade: o conceito de alteridade, com ferramentas que atentam ao aspecto ideológico da linguagem e à cultura, que aqui é considerada como sua carga principal.

\section{Referências Bibliográficas}

ADORNO, T. Esthetic theory. Minneapolis: University of Minnesota Press, 1999.

ARROJO, R. Oficina de tradução: a teoria na prática. São Paulo: Àtica, 1986.

CASTRO, F. et alii. Ven. Libro del profesor. Nivel elemental. Madri: Edelsa Grupo Didascalia, 1995.

CATFORD, F. C. Uma teoria lingüistica da tradução. Rev. Maria da Glória Novak. São Paulo: Cultrix, 1980.

DABÈNE, L. Pour une contrastivité revisité. ELA, n. 111, p. 393-478, 1998.

GRIGOLETTO, M. Leitura e funcionamento discursivo do livro didático. In: CORACINI, M. J. (Org.). Interpretação, autoria e legitimação do livro didático: língua materna e língua estrangeira. Campinas: Pontes, 1999. p. 67-77.

JACOBSON, R. Aspectos lingüísticos da tradução. In: Lingüística e comunicação. Trad. Isidoro Blikstein e José Paulo Paes. São Paulo: Cultrix, 1971. p. 63-72.

KRAMSCH, C. Context and culture in language teaching. Oxford: Oxford University Press, 1993.

LARSON, M. Meaning-based translation: a guide to cross-language equivalence. Lanham, MD: University Press of America Summer Institute of Linguistics, 1998.

MARCOS, Ma. C.; OBRA, Ma. R. Punto final. Madri: Edelsa Grupo Didascalia, 1997.

MILLER, J. H. Translation as the double production texts. In: KRAMSCH; MCCONNELL-GINER (Eds.). Text and context: cross-disciplinary perspectives on language studies. Lexington: D.C. Heath, 1992. p. 124-134.

MOUNIN, G. Osproblemas teóricos da tradução. Trad. Heloysa de Lima. São Paulo: Cultrix, 1975. 
NIDA, E. Language, culture and translation. Shanghai: Foreign Language Press, 1993.

ONG, W. J. Orality and Literacy. The Technologizing of the Word. Londres: Methuen, 1982.

ORLANDI, E. Para quem é o discurso pedagógico? Sobre Pragmática. Uberaba, p. 82-93, 1983.

- Interpretação: autoria, leitura e efeitos do trabalho simbólico. Petrópolis: Vozes, 1996.

PÊCHEUX, M. Semântica e discurso. Campinas: Editora da Unicamp, 1988. REVUZ, C. A LNM entre o desejo de um outro lugar e o risco do exílio. In: SIGNORINI, I. (Ed.). Lingua(gem) e identidade. Campinas: Mercado de Letras, 1998. p. 213-230.

SÁNCHEZ, A.; ESPINET, Ma. T.; CANTOS, P. Cumbre. Nivel medio. Madrid: SGEL-Educación, 1998.

SERRANI-INFANTE, S. Identidade e segundas línguas: as identificações no discurso. In: SIGNORINI, I. (Org.). Lingua(gem) e identidade. Campinas: Mercado de Letras, 1998, p 231-261.

STEINER, G. Après Babel: Une poétique du dire et de la traduction. Trad. Lucienne Lotringer. Paris: Edition Albin Michel, 1978.

VALDÉS, J. M. Culture in literature. In: VALDÉS, J. M. (Ed.). Culture bound. Cambridge: Cambridge University Press, 1986, p. 137-147.

VENUTI, L. The Translator's Invisibility-A History of translation. Londres/ Nova York: Routledge, 1994. 